\title{
Linezolid induced serotonin syndrome in the absence of serotonergic agent
}

Sir,

As you know, linezolid is an oxazolidinone antibiotic used widely in the treatment of methicillin-resistant Staphylococcus aureus (MRSA) or vancomycin-resistant Enterococcus (VRE) infection. Along with its antibiotic effect against gram positive cocci, it has psychotropic property with antidepressant effects through mild reversible non-selective inhibition of monoamine oxidase $(\mathrm{MAO})$ enzymes that metabolize the neurotransmitters like serotonin. ${ }^{1}$ Serotonin syndrome (SS) or serotonin toxicity occurs due to increased levels of circulating serotonin in the central and peripheral nervous system; and is characterized by mental and neuromuscular abnormalities, and autonomic hyperactivity. ${ }^{2,3}$ Linezolid administration in patients taking serotonin agonist holds a mild risk of developing SS. However, the patient being reported was not on any serotonin agonists, and yet developed SS with linezolid.

A 70 year old male, retired postman was brought with history of fever since 7 days. He was a diabetic (on gliclazide $60 \mathrm{mg}$ once daily), hypertensive (on amlodipine $5 \mathrm{mg}$ once daily) and dyslipidemic (on rosuvastatin $5 \mathrm{mg}$ at night). On examination, he was conscious, oriented and febrile $\left(100^{\circ} \mathrm{F}\right)$; with stable vitals. He had an infected ulcer over the left ankle with left lower limb cellulitis. His systemic examinations were normal. His complete blood count showed neutrophilic leucocytosis (total counts 32000 cells/cumm, N90 L10) and deranged renal functions (urea $108 \mathrm{mg} / \mathrm{dL}$, creatinine $2.75 \mathrm{mg} / \mathrm{dL}$ ). His liver functions, electrolytes, calcium and TSH were normal. ECG and chest Xray were normal, and ultrasound abdomen showed grade I renal parenchymal disease and ultrasound of left lower limb was suggestive of cellulitis with no features of deep venous thrombosis. He was started on intravenous meropenem. His HbA1c was $7.2 \%$ and sugar levels were controlled with insulin. His blood culture grew Escherichia coli which was sensitive to meropenem. However, over the next 3 days, the patient continued to be febrile. His wound swab culture grew Klebsiella which was sensitive to meropenem and linezolid. Hence, intravenous linezolid was added at $600 \mathrm{mg}$ q12h. By day 5 , patient became afebrile and his WBC counts reduced to
Access this article online

Website:

http://nepjol.info/index.php/AJMS

DOI: 10.3126/ajms.v9i4.19682

E-ISSN: 2091-0576

P-ISSN: $2467-9100$

12,600 cells/cumm. On day 7, he started developing fever spikes $\left(101^{\circ} \mathrm{F}\right)$ with profuse sweating and multiple episodes of diarrhoea. He was agitated. There was no hypoglycaemia. During the fever episodes his blood pressure shot up to 170/100 mmHg. His systemic examinations were normal. There were no signs of meningeal irritation. Mantoux test was negative. Echocardiography, repeat ECG and chest Xray were normal. Screening MRI brain and CSF analysis were also normal. Repeat blood investigations showed normal complete blood counts and liver functions, and renal parameters showed improvement (urea $52 \mathrm{mg} / \mathrm{dL}$, creatinine $2.45 \mathrm{mg} / \mathrm{dL}$ ). Urine and stool microscopy, PT/ INR and APT'T were normal, and repeat blood culture was sterile. There were no signs of thrombophlebitis, and all intravenous lines were changed. On further evaluation, he started developing these symptoms 2 days after starting linezolid. The possibility of SS was considered and linezolid was stopped, with continuation of meropenem. Within 30 hours of stoppage of linezolid, the patient became afebrile. Meropenem was given for total of 10 days, and the patient was discharged on insulin, amlodipine and rosuvastatin.

SS develops within 6 hours to 3 weeks of initiation of a medication that increases serotonin levels. These include mainly selective serotonin reuptake inhibitors, selective norepinephrine reuptake inhibitors, norepinephrine reuptake inhibitors, tricyclic antidepressants, MAO inhibitors, analgesics, dopamine agonists, isoniazid and sumatriptan. Mild SS present with tachycardia, shivering, restlessness, mydriasis, diaphoresis, tremors, myoclonus, or hyperreflexia; while moderate to severe cases manifest as hypertension, hyperthermia, bowel incontinence, clonus of the extremities, ocular clonus, rigidity, delirium, agitation, hypervigilance, pressured speech and autonomic instability. Metabolic acidosis, elevated liver enzymes, seizures, rhabdomyolysis, renal failure and disseminated intravascular coagulation are some of the consequences of SS. ${ }^{3}$ The 
diagnosis is made clinically; and there are no laboratory tests for confirmation. The Sternbach's criteria require at least 3 of the following (in the absence of neuroleptic use and other explanatory aetiologies): fever, mental abnormality, diarrhoea, agitation, myoclonus, hyperreflexia, diaphoresis, shivering, tremor and incoordination. ${ }^{2}$ Boyer's criteria require any of the following (in the presence of a serotonergic agent being administered in the past 5 weeks): tremor and hyperreflexia, spontaneous clonus, muscle rigidity and temperature $>38^{\circ} \mathrm{C}$ and clonus (ocular or inducible) with diaphoresis or agitation. The mechanism of SS is believed to involve an excess of 5-HT receptor agonism in the CNS and peripheral tissues due to high concentrations of serotonin at the synapses. ${ }^{3}$ The treatment includes removal of the offending agent, control of agitation, 5-HT2a antagonists and autonomic stabilization. ${ }^{4}$ The symptoms usually resolve within 1 to 5 days of discontinuation of the offending agent.

Not many cases of linezolid induced SS have been reported; and all these cases had the presence of a serotonergic drug. ${ }^{5-10}$ Our patient developed SS 2 days after initiation of linezolid in the absence a serotonergic drug. He had 4 features (fever, diarrhoea, diaphoresis, agitation) of Sternbach's criteria. He had a score of 7 according to Naranjo's adverse drug reaction probability scale, ${ }^{11}$ suggestive of a probable association of linezolid with SS. This case therefore highlights that linezolid can single handily induced SS.

Key words: Linezolid; Serotonin syndrome; Serotonin toxicity; Serotonergic drugs

\section{Robin George Manappallil'1, Aryasree Kakkattil ${ }^{2}$}

${ }^{1}$ Consultant, ${ }^{2}$ Resident, Department of Internal Medicine, Baby Memorial Hospital, Calicut, Kerala 673004, India

Address for Correspondence:

Dr. Robin George Manappallil,

Consultant, Department of Internal Medicine, Baby Memorial Hospital, Calicut, Kerala 673004, India. Tel: 0091-8547753396.

E-mail: drrobingeorgempl@gmail.com

\section{REFERENCES}

1. Moellering RC. Linezolid: the first oxazolidinone antimicrobial. Ann Intern Med 2003; 138(2):135-142.

2. Sternbach $\mathrm{H}$. The serotonin syndrome. Am J Psychiatry 1991; 148(6):705-713.

3. Boyer EW and Shannon M. The serotonin syndrome. N Engl J Med 2005; 352(11):1112-1120.

4. Gillman PK. The serotonin system and its treatment. J Psychopharmacol 1999; 13(1):100-109.

5. Wigen $\mathrm{CL}$ and Goetz MB. Serotonin syndrome and linezolid. Clin Infect Dis 2002; 34(12):1651-1652.

6. Thomas CR, Rosenberg M, Blythe V and Meyer WJ. Serotonin syndrome and linezolid. J Am Acad Child Adolesc Psychiatry 2004; 43(7):790.

7. Jones SL, Athan E and O'Brien D. Serotonin syndrome due to co-administration of linezolid and venlafaxine. J Antimicrob Chemother 2004; 54(1):289-290.

8. Bergeron L, Boulé $M$ and Perreault $S$. Serotonin toxicity associated with concomitant use of linezolid. Ann Pharmacother 2005; 39(5):956-961.

9. Taylor JJ, Estes LL and Wilson JW. Linezolid and serotonergic drug interactions. Clin Infect Dis 2006; 43(2):180-187.

10. Steinberg M and Morin AK. Mild serotonin syndrome associated with concurrent linezolid and fluoxetine. Am J Health Syst Pharm 2007; 64(1):59-62.

11. Naranjo CA, Busto U, Sellers EM, Sandor P, Ruiz I, Roberts EA, et al. A method for estimating the probability of adverse drugreactions. Clin Pharmacol Ther 1981; 30:239-245.

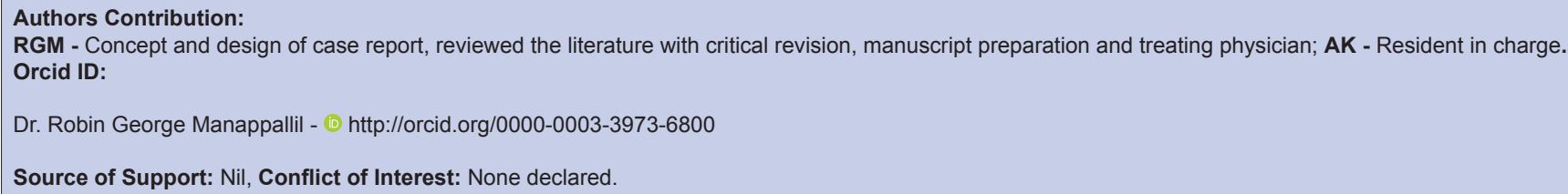

